\title{
Development of elastic lateral buckling stress for H-shaped beams with lateral-rotational braces subjected to axial force from dampers
}

\author{
Y. Yoshino ${ }^{1} \&$ Y. Kimura ${ }^{2}$ \\ ${ }^{1}$ Sendai National College of Technology, Japan \\ ${ }^{2}$ Tohoku University, Japan.
}

\begin{abstract}
H-shaped beams connected to steel dampers are subjected to compressive and tensile forces in addition to flexural moment, and then the buckling behavior of $\mathrm{H}$-shaped beams under axial force and flexural moment is more unstable than that under only flexural moment.

In this paper, the lateral buckling behavior for $\mathrm{H}$-shaped beams with the lateral bracing under the flexural moment and axial force is clarified. First, the elastic lateral buckling load of H-shaped beams is developed with the energy method, and the relationship between the lateral buckling load and the bracing rigidity is clarified. The bracing rigidities consist of the lateral and rotational rigidities. The required bracing rigidity is developed from two equations of the elastic lateral buckling load. Next, large deformation analyses are performed using the numerical analyses program ABAQUS with version 6.13, and the elasto-plastic lateral buckling behavior for $\mathrm{H}$-shaped beams with lateral and rotational bracings is presented. Finally, the lateral buckling load for H-shaped beams with the required bracing rigidity is estimated with the modified equivalent slenderness ratio, and the elasto-plastic buckling stress is compared with the buckling curve for Japanese standard code.
\end{abstract}

Keywords: lateral buckling H-shaped beam, bracing rigidity, axial force.

\section{Introduction}

When a steel moment resisting frame is subjected to seismic force, H-shaped beams carry the flexural moment. However, when dampers are applied to a moment frame, beams are subjected to axial force in addition to flexural moment. 
Then the axial force reaches $30 \%$ of the beams' yielding strength. Consequently, seismic behavior of beams of a moment frame becomes more unstable than those with no damper-

Previous research (Kimura et al. [1]) conducted static cyclic loading tests of $\mathrm{H}$-shaped beams by loading the combinations of flexural moment and axial force, and local buckling behavior and the ultimate strength were evaluated. It was shown that the ultimate strength and the plastic deformation capacity of the beams were reduced significantly, compared with those of the beams sustaining only flexural moment.

Our previous research (Kimura and Yoshino [2]) clarified the relation between the lateral buckling strength of H-shaped beams and the demands of the lateral and rotational rigidities for braces when a beam is subjected to uniform moment distribution.

In this paper, the elastic buckling load of the beams under the combination of flexural moment and axial force is developed by the energy method, and the results are verified by eigenvalue analysis. The eigenvalue analyses are also conducted for $\mathrm{H}$ shaped beams under the bending moment and axial force. Then it suggests the required bracing stiffness to restrain the buckling deformation of $\mathrm{H}$-shaped beams at the bracing points. Next, the elasto-plastic buckling strength is calculated from the large deformation analyses, and is evaluated using the buckling curve for Japanese standard code [3] with the modified slenderness ratio with the yield strength and the elastic lateral buckling load for H-shaped with lateral braces.

\section{Elastic buckling load for $\mathrm{H}$-shaped beams subjected to flexural bending moment and axial force}

\subsection{Development of elastic buckling load for $\mathrm{H}$-shaped beams subjected to flexural bending moment and axial force}

In this section, the elastic buckling load for H-shaped beams under bending moment and axial force is obtained using the energy method and eigenvalue analyses. When an H-shaped beam with brace is subjected to flexural bending moment and axial force, the upper flange of $\mathrm{H}$-shaped beam is buckled laterally and torsionally. The potential energy $U$ is expressed as the following (Kimura and Yoshino [2], Bleich [4]).

$$
\begin{aligned}
U= & \frac{1}{2} \int_{0}^{l}\left\{E I_{f} u_{1}{ }^{\prime 2}+E I_{f} u_{2}{ }^{2}+G K \beta^{\prime 2}-P_{1} u_{1}{ }^{2}-P_{2} u_{2}{ }^{\prime 2}\right\} d z \\
& +\left.\frac{1}{2} K_{u} u_{0}^{2}\right|_{z=\frac{1}{2} l}+\left.\frac{1}{2} K_{\beta} \beta^{2}\right|_{z=\frac{1}{2} l}
\end{aligned}
$$

Here, $E I_{f}$ is the flexural rigidity of each flange for H-shaped beam, $G K$ $\left(=2 G K_{f}+G K_{w}\right)$ is the torsional rigidity of H-shaped beam. $G K_{f}$ is the torsional rigidity of each flange and $G K_{w}$ is the torsional rigidity of web. $K_{u}$ is the rotational rigidity of the brace, and $K_{\beta}$ is the rotational rigidity of the brace. $\beta$ is 
the torsional angle of web. The relationship between the rotational rigidity and the flexural rigidity of braces is expressed as the equation in Fig. 1(b). $l$ is the length of $\mathrm{H}$-shaped beams. $P_{1}$ and $P_{2}$ are compressive force at beam ends $\left(P_{1}>P_{2}\right), u_{1}$ and $u_{2}$ are the lateral deformation of each flange, and $u_{0}$ is the lateral deformation at the bracing point. It is also assumed that braces are set up at upper flange, so $u_{0}$ is equal to $u_{1}$. The lateral deformation of beam is expressed as a function of the sine curves in the following. The boundary condition is simple support to strong and weak axes. When H-shaped beams is subjected to uniform moment as shown in Fig. 2, two types of load conditions are considered: one is that the stress of upper flange become compressive (designated as Type A), and the other is that the stress of upper flange become tensile (designated as Type B). Then the axial compression load and the bending moment are replaced to the axial forces of both flanges, $P_{1}$ and $P_{2}$ as shown in Fig. 3. The lateral deformation of member and web deformation are expressed as a function of the sine curves in the following.

$$
u_{1}=a_{1} \sin \frac{\pi z}{l}+a_{2} \sin \frac{2 \pi z}{l}, u_{2}=b_{1} \sin \frac{\pi z}{l}+b_{2} \sin \frac{2 \pi z}{l}
$$

Lateral displacement of the bracing, $u_{0}$, and torsional angle at the bracing point of beam, $\beta_{0}$, is expressed with the lateral deformation of flanges, $u_{1}$ and $u_{2}$ as the following, respectively.

$$
\begin{gathered}
u_{0}=u_{1} \\
\beta_{0}=\frac{u_{2}-u_{1}}{d}
\end{gathered}
$$

Substituting Eqs (2) to (4) for Eq. (1), the buckling load, $P_{c r}$, are obtained.

$$
\begin{aligned}
& P_{c r}=\frac{1}{2 p}\left[\left(P_{c r 0}+F_{\beta}\right)(p+1)+p F_{u}-\sqrt{\left\{\left(P_{c r 0}+F_{\beta}\right)(p+1)+p F_{u}\right\}^{2}}\right. \\
& \left.-4 p\left\{P_{c r 0}^{2}+P_{c r 0}\left(2 F_{\beta}+F_{u}\right)+F_{\beta}+F_{u}\right\}\right] \\
& \\
& \left.\quad \frac{P_{c r}=\frac{1}{2 p}\left[\left(P_{c r 0}+F_{\beta}\right)(p+1)+F_{u}-\sqrt{\left\{\left(P_{c r 0}+F_{\beta}\right)(p+1)+F_{u}\right\}^{2}}\right.}{-4 p\left\{P_{c r 0}^{2}+P_{c r 0}\left(2 F_{\beta}+F_{u}\right)+F_{\beta}+F_{u}\right\}}\right] \\
& \because P_{c r 0}=E I_{f}\left(\frac{\pi}{l}\right)^{2}, F_{\beta}=\frac{G K_{w}}{d^{2}}+\left(\tau_{1}+\tau_{2}\right) \frac{G K_{f}}{d^{2}}+\tau_{1} \frac{k_{\beta}^{\prime}}{d^{2}}\left(\frac{l}{n \pi}\right)^{2}, F_{u}=k_{u}^{\prime}\left(\frac{l}{n \pi}\right)^{2}
\end{aligned}
$$

Eqs (5) and (6) are the equations for the lateral buckling load of H-shaped beam with bracing on compressive flange and that on tensile flange. For these equations, the lateral deformation of $\mathrm{H}$-shaped beam at the bracing point is assumed not to be completely restrained. 


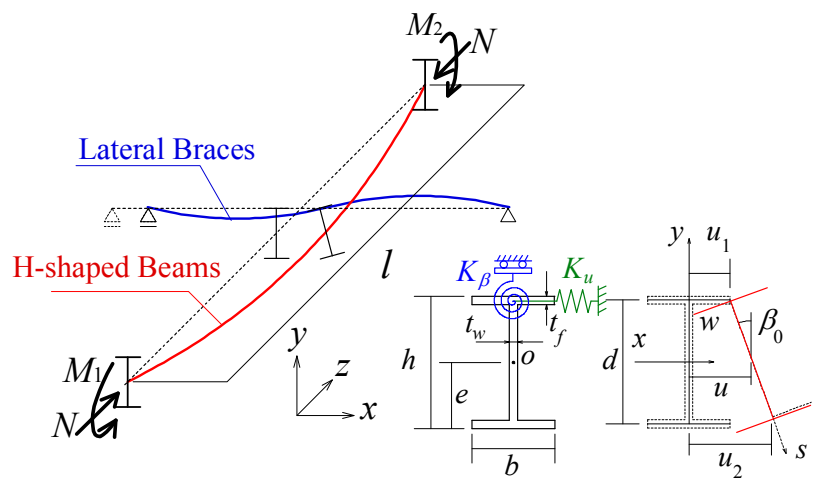

Figure 1: Lateral buckling deformation of $\mathrm{H}$-shaped beam with lateral braces on upper flange. Lateral buckling of $\mathrm{H}$-shaped beam and buckling deformation of $\mathrm{H}$-shaped beam at bracing point.
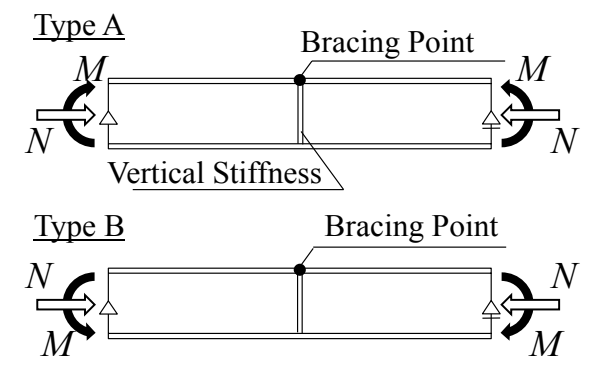

Figure 2: Loading condition.

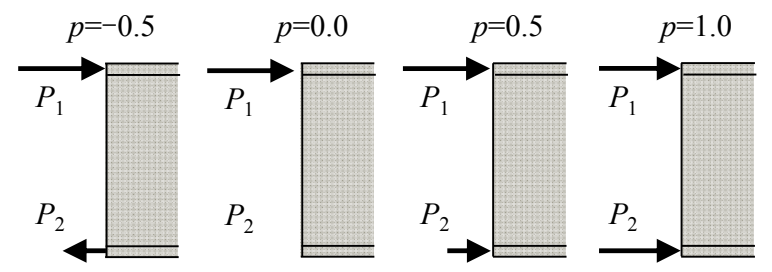

Figure 3: Ratio of the load which subjected to an upper flange and a lower flange.

$$
\begin{aligned}
& P_{c r}=\frac{1}{2 p}\left[\left\{4 P_{c r 0}+\frac{G K}{d^{2}}\right\}(p+1)-\sqrt{\left\{4 P_{c r 0}+\frac{G K}{d^{2}}\right\}^{2}(p+1)^{2}}\right. \\
& \left.-32 p P_{c r 0}\left\{2 P_{c r 0}+\frac{G K}{d^{2}}\right\}\right]
\end{aligned}
$$


On the other hand, when the lateral deformation of H-shaped beam is completely restrained at the bracing point, Eq. (7) is developed from Eq. (1) in the condition of $a_{1}=b_{1}=0$. The smaller value of $P_{c r}$ obtained from Eqs (5) and (7) is useful as the lateral buckling load for H-shaped beams with bracing on the compressive flange (Type A). Similarly, the smaller value of $P_{c r}$ obtained from Eqs (6) and (7) is useful as the lateral buckling load for H-shaped beams with bracing on the tensile flange (Type B). The lateral buckling moment, $M_{c r}$, is calculated from $P_{c r}$ obtained from Eqs (5)-(7) multiplied by the distance of both flanges, $d$.

\subsection{Effect of bracing rigidity on elastic buckling load for $\mathbf{H}$-shaped beams}

Fig. 4 presents the numerical analysis model for H-shaped beams with bracing on upper flange. ABAQUS with version 6.13 is used as numerical analyses program. The H-shaped beam and vertical stiffeners at the bracing point consists of four node shell elements, and the bracings are replaced on the lateral and rotational springs. The boundary condition is simple support to strong and weak axes. Herein, cross-sectional shapes of three kinds are adopted as shown in Table 1. Then each cross-sectional shape is selected as the ratio of flange width to web depth, $b / h=0.33,0.5,0.77$, respectively. For these examinations, a 50-200 lateral rigidity ratio $k_{u} /\left(E I_{f} / l^{3}\right)$ and a $2.5-5.0$ rotational rigidity ratio $k_{\beta} /\left(G K_{f} / d\right)$ are adopted.

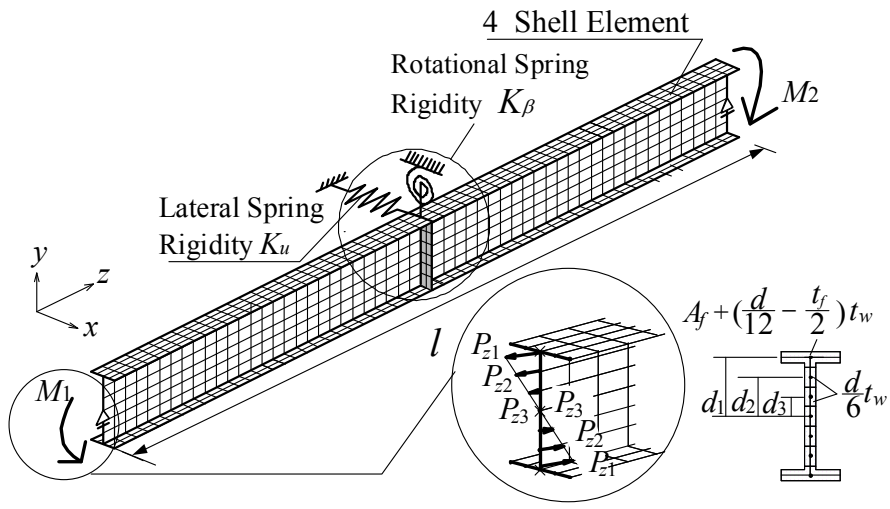

Figure 4: Numerical analysis model.

Table 1: Cross sectional shape of H-shaped beams.

\begin{tabular}{|c|c|c|c|c|c|c|c|}
\hline$B / H$ & $H$ & $\times$ & $B$ & $\times$ & $t_{w}$ & $\times$ & $t_{f}$ \\
\hline \hline 0.77 & 390 & $\times$ & 300 & $\times$ & 10 & $\times$ & 16 \\
\hline 0.5 & 500 & $\times$ & 250 & $\times$ & 9 & $\times$ & 16 \\
\hline 0.33 & 600 & $\times$ & 200 & $\times$ & 11 & $\times$ & 17 \\
\hline
\end{tabular}


Fig. 5 presents the relation between the elastic buckling stress, $\sigma_{c r}$, for $\mathrm{H}$ shaped beams with braces on upper flange and the ratio of axial compressive forces on upper and lower flanges, $p\left(=P_{2} / P_{1}\right)$. The curves show the buckling stress, $P_{c r} / A_{1}$, obtained from Eqs (5)-(7). Here, $A_{1}=A_{f}+A_{w} / 6 ; A_{f}$ is the area of compressive flanges and $A_{w}$ is the web area of H-shaped beams. The symbols show the eigenvalue analyses results. The beams with $b / h=0.5, K_{u} /\left(E I_{f} / l^{3}\right)=50$ and $K_{\beta} /\left(G K_{f} / d\right)=5$ are selected. The dots $\mathbf{\nabla}$ is the points to change from the buckling mode lateral and torsional deformed at the brace point to that perfectly fixed at the brace point. The buckling curves obtained from Eqs (5)-(7) and the symbols of the eigenvalue analyses results are very fitting well. Eqs (5)-(7) can be applied to estimate the elastic buckling stress of $\mathrm{H}$-shaped beams under bending moment and axial force. The black and grey curves show the buckling stress for Type A and Type B, respectively. The buckling stress for Type A and B are almost equal at $p=0$. The smaller the ratio of axial compressive forces on both flanges, $p$ becomes, the larger the buckling stress becomes. As the slenderness ratio becomes larger, $p$ at the dots $\boldsymbol{\nabla}$ becomes larger.

Fig. 6 shows the relation between the lateral rigidity ratio and the rotational rigidity ratio for the required rigidity. The beams with $b / h=0.5$ and $\lambda_{1}=120$ is selected, and the parameters are the ratio of axial compressive forces on upper and lower flanges, $p$ and the loading condition. The curves represent the case for the bracing rigidity changed from the buckling mode laterally and torsionally deformed at the brace point to that perfectly fixed at the brace point. The black curves represent the cases of Type A, and the gray curves represent the cases of Type B. The curves for Type A are drawn from Eq. (5) and Eq. (7). The curves for Type B are drawn from Eq. (6) and Eq. (7). The symbols of horizontal axis, $\Delta$ and $\Delta$ represent the minimum required rotational rigidity, $K_{\beta 0}$, at $K_{u}=\infty$. The symbols of vertical axis, - and - denotes the minimum required lateral rigidity, $K_{u 0}$, at $K_{\beta}=\infty$.

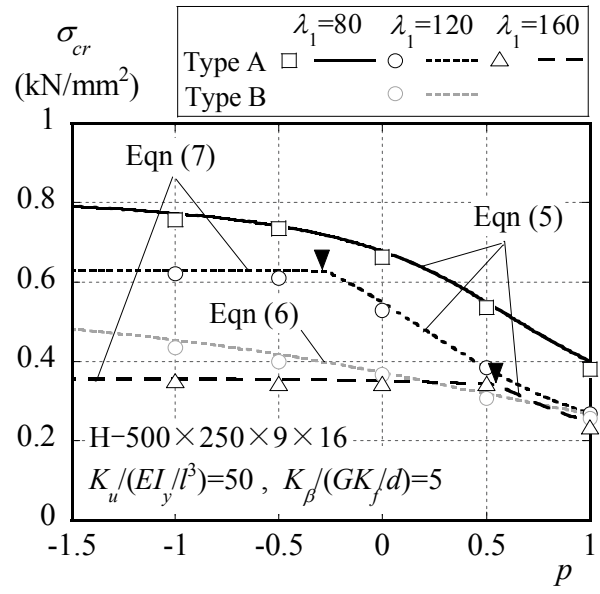

Figure 5: Lateral buckling stress of H-shaped beams.

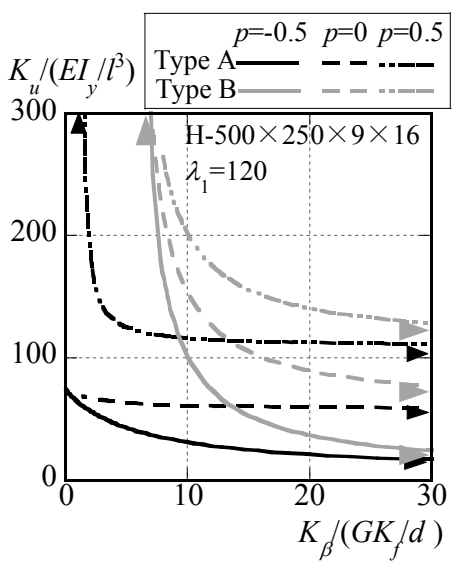

Figure 6: Required rigidity of H-shaped beams. 
The buckling load for less than $K_{u 0}$ or $K_{\beta 0}$ never reaches that perfectly fixed at the brace point, even though the other rigidity becomes larger. The smaller the ratio of axial forces, $p$ becomes, the smaller the required rigidity becomes. For $p=0$ and $p=-0.5$, the buckling deformation at the brace point is perfectly fixed, even though $K_{u}$ is equal to 0 . If the rotational rigidity is smaller than the minimum required rotational rigidity, or if the lateral rigidity is smaller than the minimum required lateral rigidity, then no matter how large the other rigidity is, the buckling load does not reach that perfectly fixed at the brace point.

\section{Elasto-plastic buckling strength for $\mathrm{H}$-shaped beams with braces subjected to flexural bending moment and axial force}

\subsection{Elasto-plastic buckling behaviour for H-shaped beams}

In this section, elasto-plastic buckling behavior for $\mathrm{H}$-shaped beams with braces subjected to bending moment and axial force is confirmed using the elastoplastic large deformation analyses (FEM). Then the elasto-plastic buckling stress for these members is evaluated with the buckling curve for Japanese and U.S. design codes using the modified slenderness ratio.

FEM analyses are performed to investigate the elasto-plastic buckling behavior for $\mathrm{H}$-shaped beam. The analyses model and boundary condition are the same as those used for the eigenvalue analyses models described in the previous section. Material properties for H-shaped beams are presented in Table 2. The initial impressions of the lateral displacement, $u$, the torsional angle, $\beta$ are described as the function of sine curves in the following.

$$
\begin{gathered}
u=\frac{l}{2500} \sin \frac{\pi z}{l}+\frac{l}{10000} \sin \frac{2 \pi z}{l} \\
\beta=\tan ^{-1}(u / d)
\end{gathered}
$$

Table 2: $\quad$ Materials properties of H-shaped beams.

\begin{tabular}{cccc|}
\hline$E\left(\mathrm{~N} / \mathrm{mm}^{2}\right)$ & $\sigma_{y}\left(\mathrm{~N} / \mathrm{mm}^{2}\right)$ & $\sigma_{u}\left(\mathrm{~N} / \mathrm{mm}^{2}\right)$ & $E_{s t}\left(\mathrm{~N} / \mathrm{mm}^{2}\right)$ \\
\hline 210000 & 294 & 436 & 2740 \\
\hline
\end{tabular}

Fig. 7 presents the required rigidity curves for Type B and the selected sample point of ${ }_{\mathrm{B}} k_{0}$ for elasto-plastic analyses models with $b / h=0.33, \lambda_{1}=160$ and $p=-1.0$. The black of surface of required rigidity are represented as the function, $k_{0}$, obtained from Eqs (5) and (7) for Type B.

There is no required rigidity at $K_{u}=0$ for Type B. Therefore the lateral rigidity for $\mathrm{H}$-shaped beams is selected by Japanese design code [3]. The lateral rigidity ratio, $K_{u} /\left(E I_{y} / l^{3}\right)=131$ is constant irrespective of the cross-sectional shapes and slenderness ratio. The intersection point of the black surface, $k_{0}$ and $K_{u} /\left(E I_{y} / l^{3}\right)=131$ is selected as the required rigidity, ${ }_{\mathrm{B}} k_{0}$. 


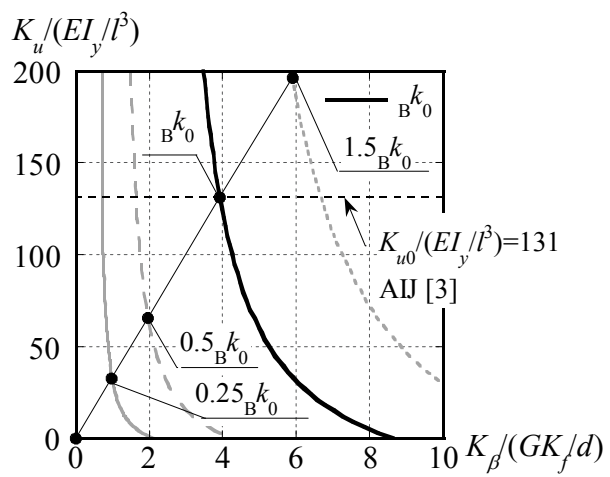

Figure 7: Lateral and rotational rigidity for elasto-plastic analyses models.

The ratio of bracing rigidities between $0.25 k_{0}$ and $1.5 k_{0}$ is selected for numerical analyses. For example, the coordinates on the curve of $0.5 k_{0}$ are half of the coordinates on the curve of $k_{0}$. Thereby the values of $K_{u} /\left(E I_{y} / l^{3}\right)$ and $K_{\beta} /\left(G K_{f} / d\right)$ for $0.5 k_{0}$ means half of those for $k_{0}$, respectively.

Fig. 8 shows the elasto-plastic buckling behavior for H-shaped beams with lateral braces subjected to bending moment and axial force. The beams with $b / h=0.33, \lambda_{1}=160$ are selected. The parameter are the magnitudes of the required rigidity and the ratio of axial compressive forces on upper and lower flanges, $p\left(=P_{2} / P_{1}\right)$. Figs $8(\mathrm{a})-8(\mathrm{c})$ present the ratio of the bending load to the yielding load $P_{2} / P_{y}$, the lateral displacement $u / l$, the torsional angle $\beta$. Herein, $P_{2}$ is the load of low flange.

The symbols $u$ and $\beta$ in Figs 8(b) and 8(c) represent the displacement at the center of beams. The maximum load with $k_{0}$ is about $10 \%$ larger than that with $0.5 k_{0}$, because the braced rigidity for $k_{0}$ is twice larger than that for $0.5 k_{0}$. In Figs. $8(\mathrm{~b})$ and $8(\mathrm{c})$, lateral displacement and torsional angle curves for $0.5 k_{0}$ are larger than those for $k_{0}$.

Fig. 9 presents the concept of the equivalent sectional area to carry the axial compressive load, $P_{1}$ and $P_{2}$. The critical load $P_{c r}$ is divided to the axial compressive force, $N_{c r}$ and the bending moment, $M_{c r}$ as shown in Fig. 9(a) and 9(b), and the flange's axial stresses issued from the axial compressive force, $\sigma_{c r N}$ and the bending moment, $\sigma_{c r}$ are obtained, respectively. The flange's axial stress in Fig. 9(c) is equal to the sum of the axial compressive stresses, and from the equilibrium equation, the equivalent area on larger compressive flange is expressed in the following.

$$
A_{p}=\left(\frac{d}{Z} \frac{1-p}{2}+\frac{1+p}{A_{0}}\right)^{-1}
$$

The equivalent sectional area, $A_{p}$ consists of the flange area, $A_{f}$ and the part of the web area, $\alpha A_{w}$. $\alpha$ is varied from $1 / 6$ to $1 / 2$ by the axial force ratio between the upper and lower flanges as shown in Fig. 10, and the curves for three kinds of sectional member are almost identical. 


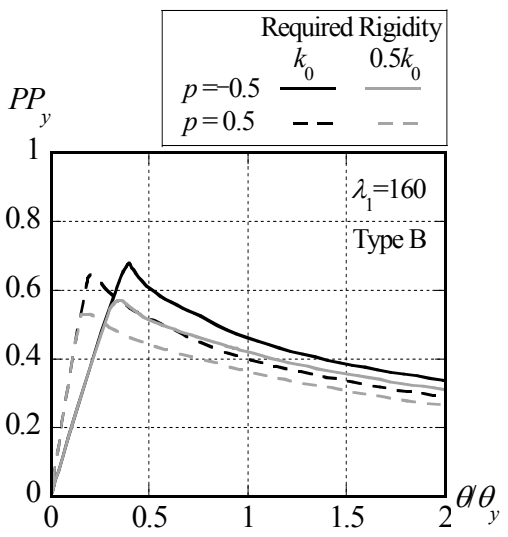

(a)

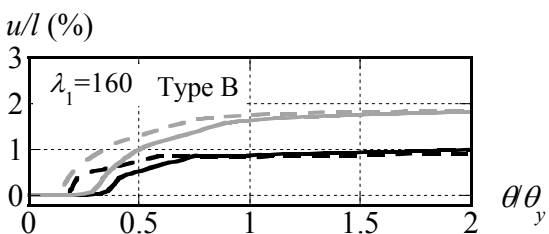

(b)

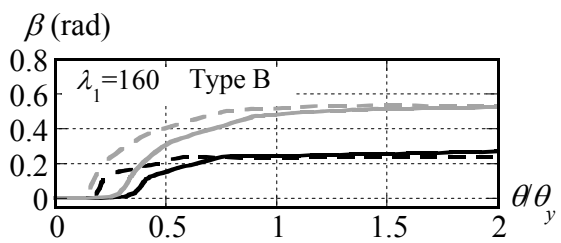

(c)

Figure 8: Elasto-plastic lateral buckling behavior for $\mathrm{H}$-shaped beams. (a) Buckling load and rotation; (b) Lateral deformation and rotation; (c) Torsion and rotation.

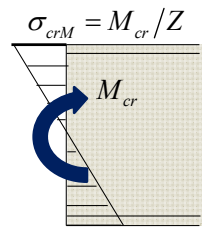

(a) Flexual Stress $\sigma_{c r M}$

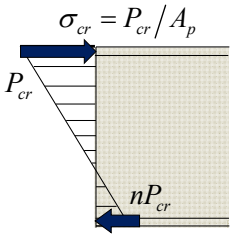

(c) Bucklingl Stress $\sigma_{c r}$

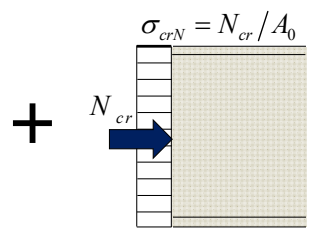

(b) Axial Stress $\sigma_{c r N}$

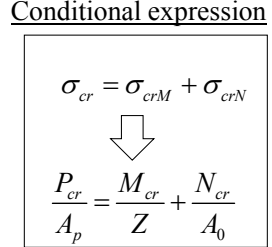

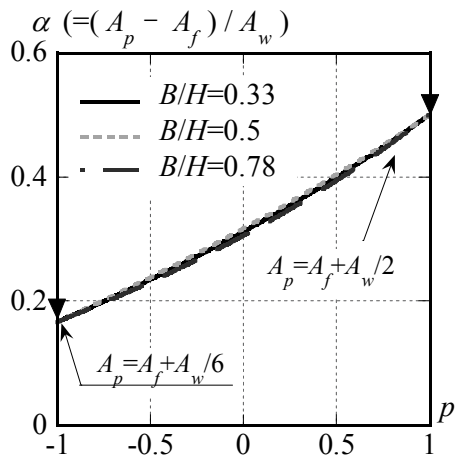

Figure 9: Concept of equivalent sectional Figure 10: Rate of sectional area area. of web to equivalent sectional area. 


\subsection{Estimation of elasto-plastic buckling stress for $\mathrm{H}$-shaped beams with continuous braces}

Fig. 11 portrays the relation between the elasto-plastic buckling stress, $\sigma_{c r} / \sigma_{y}$ for $\mathrm{H}$-shaped beams with braces and modified equivalent slenderness ratio, $\lambda_{c}$. In fact, $\lambda_{c}$ is shown as the following in the Japanese design code [3].

$$
\lambda_{c}=\sqrt{P_{y} / P_{c r}}
$$

Here, $P_{y}$ is the yield load and $P_{c r}$ is Euler's buckling load. When H-shaped beams with brace are subjected to bending moment and axial force, $P_{c r}$ in Eq. (10) is replaced on the buckling load obtained from Eqs (5)-(7). $P_{y}$ is the yield stress multiplied by the equivalent area, $A_{p}$ as follows.

$$
\overline{\lambda_{b}}=\sqrt{\overline{P_{y}} / P_{c r}} \quad \because \overline{P_{y}}=\sigma_{y} A_{p}
$$

This equivalent slenderness ratio in Eq. (12), $\overline{\lambda_{b}}$ is called the modified equivalent slenderness ratio.
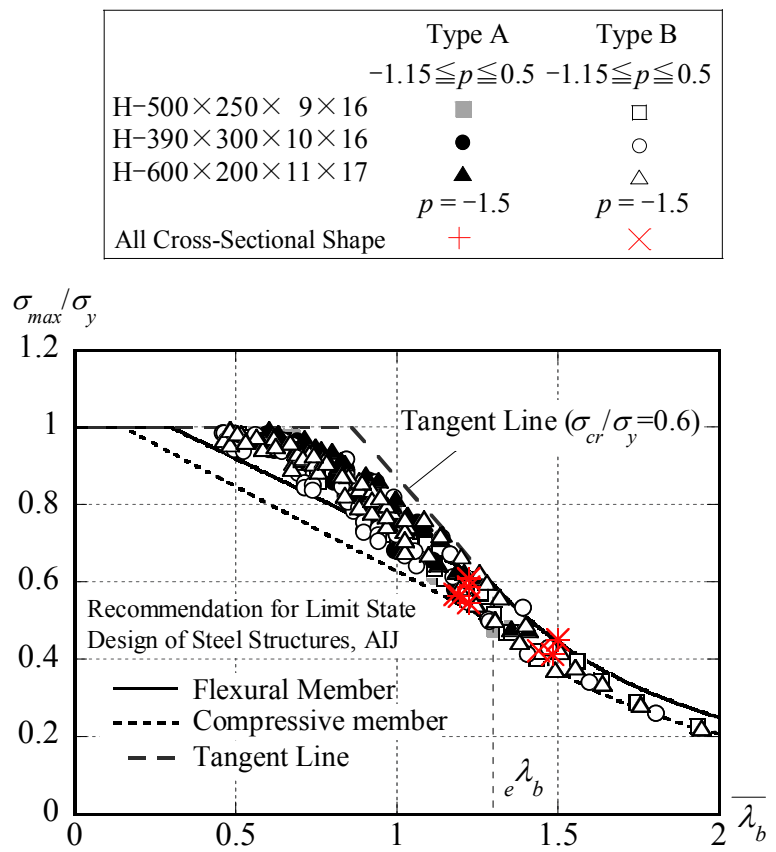

Figure 11: Lateral buckling stress of $\mathrm{H}$-shaped beams with braces.

These curves in Fig. 11 are the buckling stress curves for Japanese design codes [3], and the tangent line of Euler's buckling curve from $\sigma_{c r} / \sigma_{y}=0.6$. The symbols show results of numerical analyses. The symbols constantly exceed 
the buckling stress curve for bending moment in the range of $\bar{\lambda}_{b}>0.9$, and these constantly exceed that for compressive forces in the range of $\bar{\lambda}_{b} \leq 0.9$. Also the symbols distributed as the upper-bound for the tangent line of $\sigma_{c r} / \sigma_{y}=0.6$. Consequently, the elasto-plastic buckling stress for H-shaped beams subjected to bending moment and axial force can be evaluated approximately by the buckling curves in Japanese design codes [3] using the modified equivalent slenderness ratio.

\section{Conclusions}

This paper evaluated the effect of the lateral and rotational braces on lateral buckling stress for $\mathrm{H}$-shaped beams subjected to bending moment and axial force. Results show the following.

1. The elastic lateral buckling load for H-shaped beams under the combination of flexural moment and axial force is obtained from Eqs (5)-(7). When the upper flange's compressive load is larger than the lower flange's one, the smaller value obtained from Eqs (5) and (7) is applicable as the elastic buckling load for these beams. When the upper flange's compressive load is smaller than the lower flange's one, the smaller value obtained from Eqs (6) and (7) is applicable as the elastic buckling load for these members.

2. It is shown that the lateral deformation of upper flange is completely restrained at the bracing point, when the braces with the proposing required rigidities are set to the H-shaped beam.

3. The approximate elasto-plastic buckling stress for H-shaped beams with the lateral and rotational braces can be estimated using the equations in the Japanese design codes with the modified equivalent slenderness ratio in Eq. (9).

\section{References}

[1] Kimura, Y., Yamanishi, T. and Kasai, K. (2013). Cyclic Hysteresis Behavior and Plastic Deformation Capacity for H-Shaped Beams on Local Buckling Under Compressive and Tensile Forces, AIJ. 78, 689, 1307-1316 (in Japanese).

[2] Kimura, Y. and Yoshino, Y. (2011). Required Bracing Capacity on Lateral Buckling Strength for H-shaped beams with bracings, AIJ. 76, 670, 21432152 (in Japanese).

[3] Architectural Institute of Japan, AIJ (1998). Recommendation for Limit State Design of Steel Structures buckling (in Japanese).

[4] Bleich, F. (1952). Buckling Strength of Metal Structures, McGraw-Hill Book Co., 142-147. 